\title{
Recent Advances in Self-Powered Electrochemical Systems
}

\author{
Linglin Zhou, ${ }^{1,2}$ Di Liu, ${ }^{1,2}$ Li Liu, ${ }^{1,2}$ Lixia He, ${ }^{1,2}$ Xia Cao $\mathbb{D}^{1},{ }^{1}$ Jie Wang $\mathbb{D},{ }^{1,2}$ \\ and Zhong Lin Wang ${ }^{1,3}$ \\ ${ }^{1}$ Beijing Institute of Nanoenergy and Nanosystems, Chinese Academy of Sciences, Beijing 100083, China \\ ${ }^{2}$ College of Nanoscience and Technology, University of Chinese Academy of Sciences, Beijing 100049, China \\ ${ }^{3}$ School of Materials Science and Engineering, Georgia Institute of Technology, Atlanta, GA 30332, USA
}

Correspondence should be addressed to Xia Cao; caoxia@binn.cas.cn, Jie Wang; wangjie@binn.cas.cn, and Zhong Lin Wang; zhong.wang@mse.gatech.edu

Received 28 December 2020; Accepted 17 February 2021; Published 12 March 2021

Copyright (C) 2021 Linglin Zhou et al. Exclusive Licensee Science and Technology Review Publishing House. Distributed under a Creative Commons Attribution License (CC BY 4.0).

\begin{abstract}
Electrochemistry, one of the most important research and production technology, has been widely applicated in various fields. However, the requirement of external power source is a major challenge to its development. To solve this issue, developing selfpowered electrochemical system (SPES) that can work by collecting energy from the environment is highly desired. The invention of triboelectric nanogenerator (TENG), which can transform mechanical energy into electricity, is a promising approach to build SPES by integrating with electrochemistry. In this view, the latest representative achievements of SPES based on TENG are comprehensively reviewed. By harvesting various mechanical energy, five SPESs are built, including electrochemical pollutants treatment, electrochemical synthesis, electrochemical sensor, electrochromic reaction, and anticorrosion system, according to the application domain. Additionally, the perspective for promoting the development of SPES is discussed.
\end{abstract}

\section{Introduction}

Electrochemistry, which refers to the interrelation of electrical and chemical effects, has play a crucial part in the sustainable advancement and innovation of industrial processes, including chemical industry, medicine, materials, energy, metal corrosion, and environmental science [1-5]. Electrochemical process highly depends on external power supply, which aggravates the crisis of energy shortage and environmental pollution problems in modern society. To solve these issues, developing a self-powered electrochemical system (SPES), which can operate by integrating electrochemical system with energy harvesting technology for collecting energy form environment [6-10], is one of the promising approaches. On account of the sufficient availability of mechanical energy, converting it from environment to electricity has aroused broad attentions. Recently, many technologies that extract mechanical energy have been reported, such as electromagnetic generator [11] and piezoelectric nanogenerator [6]. However, the low energy conversion efficiency of electromagnetic generator at low frequency and low output power of piezoelectric nanogenerator limit their practical application.

Based on the conjunction of triboelectrification and electrostatic induction, triboelectric nanogenerator (TENG), also known as Wang generators, was invented to extract energy from a variety of ambient mechanical motions, like sound, water waves, and mechanical vibration [12-15]. TENG exhibits many unique advantages including light structural simplicity, diverse materials options, and high conversion efficiency [16]. Moreover, TENG has demonstrates its potential applications in micro/nano power sources $[17,18]$, selfpowered (SP) sensors $[19,20]$, large-scale blue energy [21, $22]$, and direct high voltage power sources [22, 23]. To promoting the practical application of TENG, large researches have been focused on improving its output performance via investigating the basic principles [24], enhancing the surface charge density [25-30], and power management [31-33]; thus, $500 \mathrm{~W} \cdot \mathrm{m}^{-2}$ of area power density and $>50 \%$ of conversion efficiency have been achieved, respectively [34]. In view of these advantages, TENG can be served as a promising alternatively energy harvesting power source to integrate 


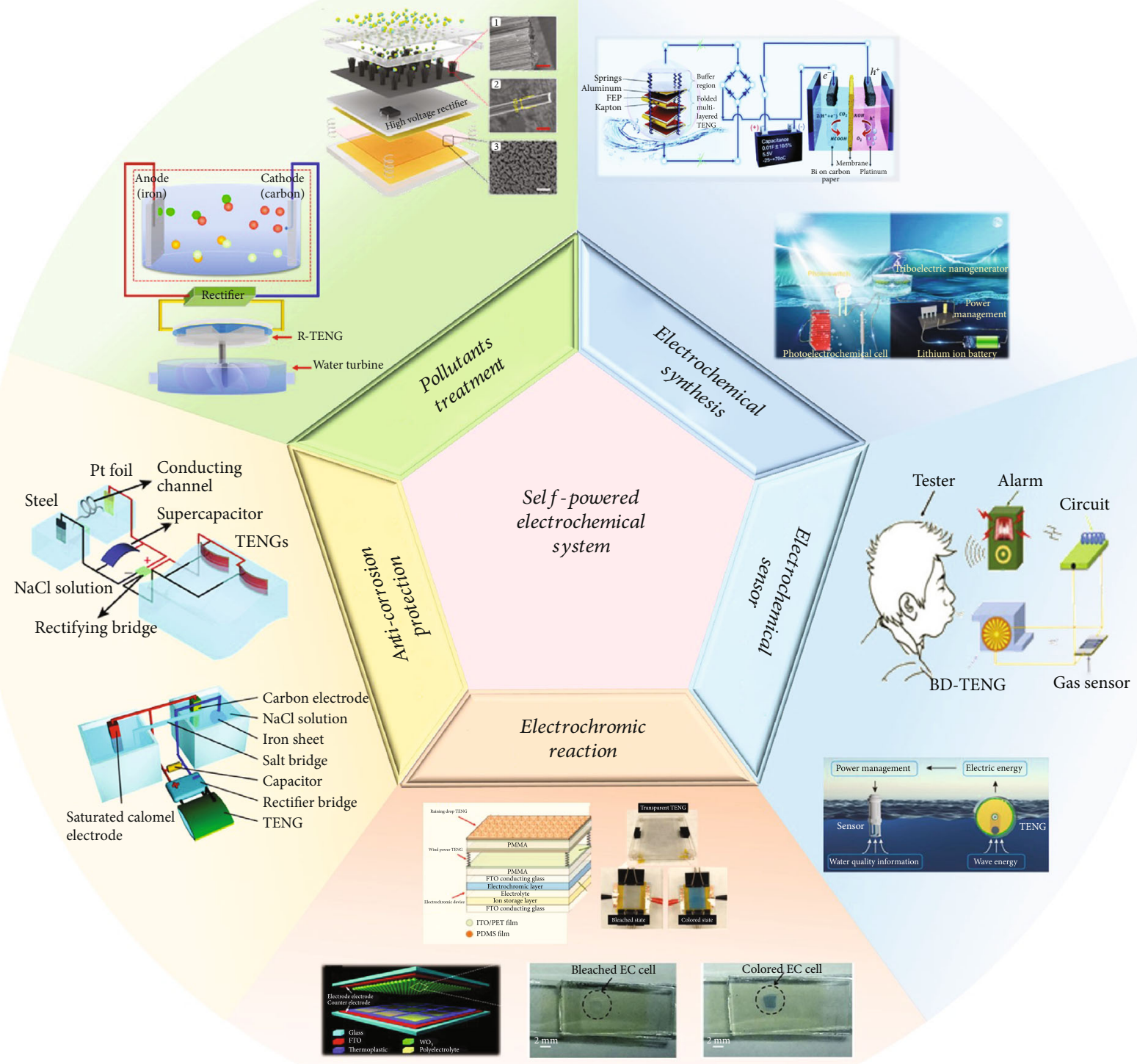

FIgURE 1: Diverse applications of self-powered electrochemical system. SP electrochemical system for pollutants treatment [50, 63], electrochemical synthesis [70,78], electrochemical sensor [89, 95], electrochromic reaction [99, 100], and anticorrosion protection [112, 113].

with electrochemistry for electrochemical operation. Currently, many SPESs based on TENG have been developed, which will be elaborated in further detail below. Compared with traditional electrochemical systems, SPESs can drive the electrochemical process without the external power supply, which largely promote the sustainable development of electrochemical systems.

Here, the recent progresses and practical applications of SPES based on TENGs are summarized. According to the application, the SPES is classified into five major applications including pollutants treatment, electrochemical synthesis, SP sensors, electrochromic reaction, and anticorrosion system (Figure 1), respectively. Additionally, perspectives and challenges for promoting the development of SPES are proposed.
We would very much like that this paper will significantly advance the development of TENG in the electrochemistry field and offer a direction for future research in SPES.

\section{Self-Powered Electrochemical System}

SPES is developed from the combination of electrochemistry and TENG technology, where electrochemical process can operate by TENG that produces electrical power from the ambient environment. As a mechanical energy harvester, the recent development of TENG has been reviewed from the mechanism to the potential applications $[35,36]$. Classified according to the motion direction and the capacitance change, four basic working modes of TENG were proposed 
since its first invention in 2012 [37-41], which can be divided into two types including contact-separation-type and slidingtype TENG. Driven by external force, repetitive changing of the distance between two planes and the size of contacting area will cause the variation of capacitance of TENG, resulting in the potential difference in two electrodes. For balancing the potential difference, electrons will flow back and forth in external circuit and thus generates an alternative current output. For driving the electrochemical process, a direct current is always employed in the current researches about SPES, where the alternative current output of TENG should be firstly transformed to direct current output by using rectifying device and then drive the electrochemical process.

The fundamental physics mechanism of TENG derives from Maxwell's displacement current [42, 43], which is defined as

$$
J_{D}=\frac{\partial \boldsymbol{D}}{\partial t}=\varepsilon \frac{\partial \boldsymbol{E}}{\partial t}+\frac{\partial \boldsymbol{P}_{s}}{\partial t}
$$

where $J_{D}, D, P_{S}$, and $E$ are the total displacement current density, the electric displacement vector, the polarization field, and the electric field, respectively. The displacement current $\left(I_{D}\right)$ is a surface integral of $J_{D}$ :

$$
I_{D}=\int J_{D} d S=\int \frac{\partial D}{\partial t} d S=\frac{\partial}{\partial t} \int \nabla \cdot D d r=\frac{\partial}{\partial t} \int \rho d r=\frac{\partial Q}{\partial t} a,
$$

where $S, \rho$, and $Q$ are the medium surface, the distribution of free charges, and the total free charges on the electrode. According to the equation, the output loop of TENG contains two portions, including the internal circuit in TENG that is controlled by displacement current and the observed current in the external circuit. In summary, the core of physics for current production is the internal driving force of $\partial \boldsymbol{P}_{s} / \partial t$, which is named as the Wang term in the displacement current [43], and the external manifestation of displacement is the observed capacitive conduction current in external circuit.

\section{Applications of Self-Powered Electrochemical System}

3.1. Pollutant Treatment. Owing to the modern industrial activities, an alarming increase of toxic pollutants in the environment caused by human activities has brought to serious environmental issue, such as water pollution and air pollution, which is the most important environmental factor of disease and premature death in the world today [44]. For the growing threat of environmental pollutants, high efficiency pollutant treatment strategy is highly desired to ensure clean environment and human health. Due to high removal efficiency, great versatility, high amenability, and excellent environmental compatibility, electrochemical technology has been extensively developed as a promising method for environmental pollutants treatment $[45,46]$. However, the requirement of an external power supply has become the major challenge for the practical application of electrochemical technology. Due to the invention of TENG by collecting mechanical energy from environment, SPES has been proposed as a candidate for environment treatment [47]. Furthermore, with the gradually enhanced output performance of TENG, many efforts have been devoted for removing environmental pollutants from water and air.

Water pollutants are mainly composed of inorganic, organic, and biological contaminants. As one of the most toxic pollutants, heavy metal ions can biologically accumulate upon cumulative exposure, which is not biodegradable, and thus threaten human health via the entire food chain [48]. Aiming to remove heavy metal ions from environment, $\mathrm{Li}$ et al. reported a water-driven TENG to extract the kinetic energy from wastewater flow, which used to drive electrochemical reaction for $\mathrm{Pb}^{2+}$ and $\mathrm{Cu}^{2+}$ removal [49]. By using the integrated SP system, $97.4 \%$ of the two metal ions was removed from the wastewater in $100 \mathrm{~min}$. Through comparing the electrochemical property of $\mathrm{Cr}(\mathrm{VI})$ driven by continuous DC (CDC) and pulsed DC (PDC), Zhou et al. confirmed an enhanced efficiency of removing $\mathrm{Cr}(\mathrm{VI})$ under PDC than that of CDC due to the better utilization of ferrous ion, the lower electrode passivation, and the higher ion diffusion rate during the reaction process [50]. Therefore, they proposed an SPES based on TENG with PDC output for improving the electrochemical performance of heavy metal ion treatment. The structures of the rotary TENG and SPES are shown in Figures 2(a) and 2(b). The charge consumption under different power supply is shown in Figure 2(c). Under equal charges consuming with $0.048 \mathrm{C}$, the removal efficiency driven by PDC was increased by $53.5 \%$ compared to that of CDC.

Organic pollutants in water have also drawn wide concern because of the most toxic and potentially carcinogenic; therefore, large efforts have been devoted to removing organic pollutants from wastewater. SP electrooxidation is the common process for organic pollutants treatment by using the produced chlorine and hypochlorite. Li et al. proposed a unique SP electrooxidation system to phenol removal via creatively employing $\beta$-cyclodextrin to increase triboelectrification [51]. In the condition of wastewater wave with velocity of $1.4 \mathrm{~m} \mathrm{~s}^{-1}$ and initial concentration of phenol with $80 \mathrm{mg} \mathrm{L}^{-1}, 90 \%$ of the phenol was removed by the generated power in $320 \mathrm{~min}$. Gao et al. reported a free-standing mode TENG integrated with electrocatalytic technology for degrading 4-aminoazobenzene [52]. With a commercial aluminum panel as stator, the power density reached up to $2.28 \mathrm{~W} \mathrm{~m}^{-2}$. Driven by the TENG, 4-aminoazobenzene can be electrochemical degradation to small molecule polymers by sensibly adjusting the potentials of electrochemical oxidation. By using the sponge to improve the contact intimacy and precharge injection to increase the surface charges in the dielectric film, Gao et al. improved the power density of a multilayer linkage TENG to $7.4 \mathrm{~W} \mathrm{~m}^{-2}$, which was used to drive electrochemical catalysis for degrading methyl red [53]. Powered by the multilayer linkage TENG, the degradation percentage of methyl red was almost $100 \%$ after $160 \mathrm{~min}$, with a color change from red to colorless. Additionally, Chen et al. fabricated an SP multifunctional system which can 


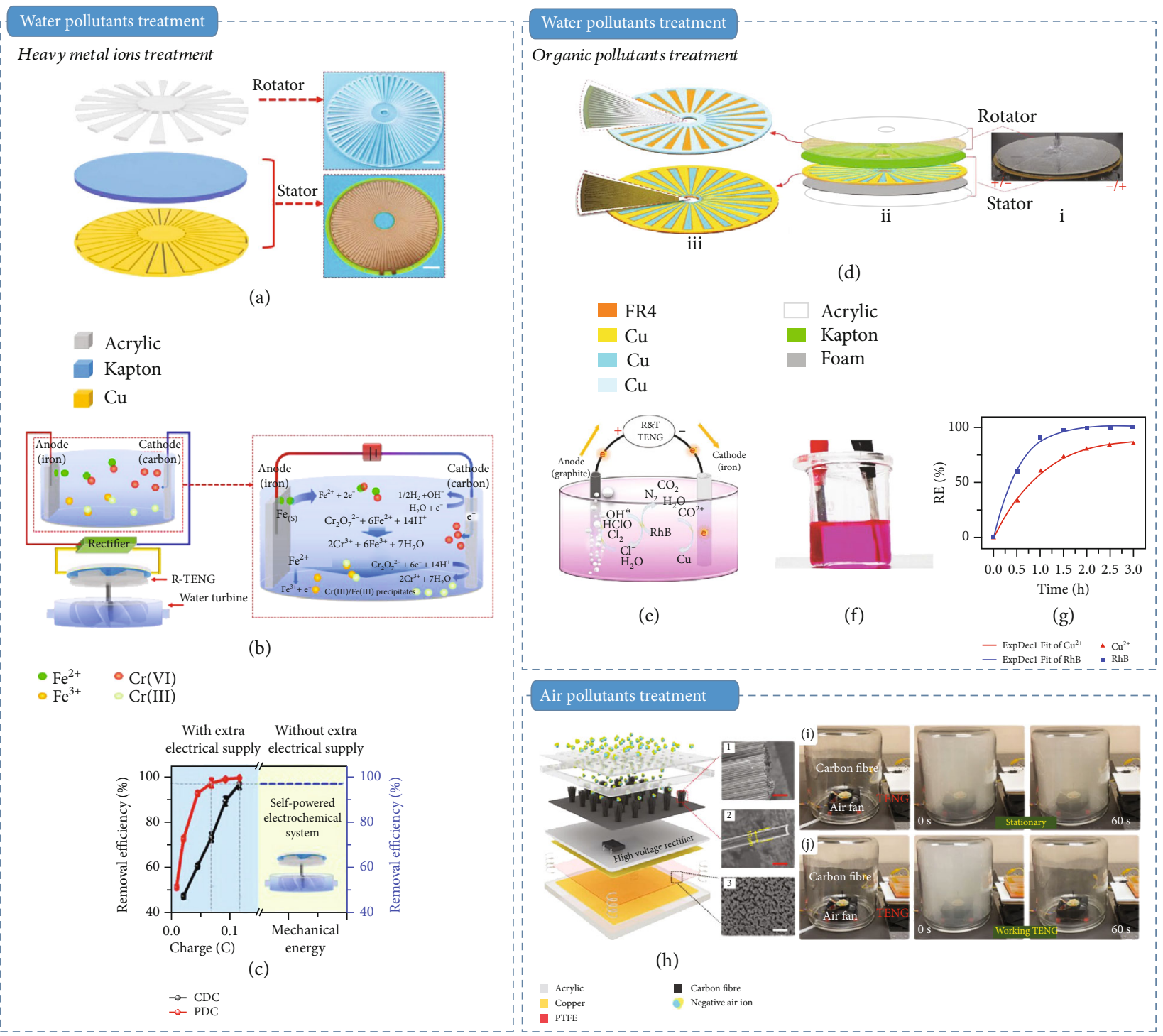

FIGURE 2: Self-powered electrochemical pollutant treatment: (a) sketch map of rotary TENG; insert displays the pictures of stator and rotator; (b) SP system for removing Cr(VI) powered by rectified TENG; (c) the comparison of charge consumption for Cr(VI) removal under different conditions [50]; (d) sketch map of the rotary TENG; (e, f) the mechanism image and optical image of SP electrochemical system for cupric ions and rhodamine B removal; (g) removal efficiency of cupric ions and rhodamine B [54]; (h) sketch map of the triboelectric negative air ion generator; $(i, j)$ the contrast photographs of the smog cleaning by the MSNG in stationary conditions (i) and with the FS-TENG conducting at $0.25 \mathrm{~Hz}(\mathrm{j})[63]$.

simultaneously realize heavy metal ions and organic pollutant removal driven by a rotary TENG [54]. The illustrations of the rotary TENG and system are shown in Figures 2(d) and 2(e). After rectifying, $100 \%$ of rhodamine B and $97.3 \%$ cupric ion were removed after 3 hours treatment (Figures 2(f) and 2(g)). Besides, Yang et al. reported an SP electrocatalytic system containing a hybrid energy cell including TENG to degrade methyl orange, which can further achieve a higher performance [55]. In this system, the generated energy can directly power the electro-degradation of methyl orange or store in an energy storage unit before utilizing for methyl orange treatment, where the removal efficiency of methyl orange reached $80 \%$ after $144 \mathrm{~h}$.

Owing to merits of environmentally friendly and particularly efficient, SP electrochemical advanced oxidation system such as SP electro-Fenton process is proposed to removing organic pollutants from the water [56-58]. Feng et al. integrated a rotary TENG with an electrochemical cell to build an SP electro-Fenton system to remove dyes [59]. In this work, a modified graphite felt cathode was used to produce $\mathrm{H}_{2} \mathrm{O}_{2}$ along with $\bullet \mathrm{OH}$, and a platinum sheet anode was applied to generate active chlorine, which can be used to oxidize organic pollutants. Through power management, the electric power of integrated rotary TENG improved by 3.5 times for driving electrochemical reaction. Driving by wind flow at the speed of $6.2 \mathrm{~m} \mathrm{~s}^{-1}$, the SP electro-Fenton system can efficiently degrade dyes without aerating oxygen, and a removal efficiency of $87.5 \%$ within $120 \mathrm{~min}$ was achieved. Combining with 3D printing techniques, Tian et al. prepared a $3 \mathrm{D}$ printed elastic TENG to form an SP electro-Fenton 
system for removing methylene blue, where $97.0 \%$ of methylene blue was removed within $140 \mathrm{~min}$ [60].

Biological contaminants such as harmful bacteria and algal blooms caused by uncontrolled discharge of wastewater are the other kinds of water pollutants. Jiang et al. reported an SP electrochemical water treatment system on the basis of an arch-shaped TENG for cleaning sterilization and algae in wastewater [61]. By extracting water wave energy, most of the bactericidals and algae treatment were cleaned by the produced $\mathrm{Cl}_{2}$ and reduced graphene oxide. By using SPES, high removal efficiencies for three model bacteria and mixed marine algae were achieved.

Air pollution including fine air particulate matter (PM) and gaseous pollutants is a crucial toxicant caused by human activities. Generally, air pollution exposure is related to lots of chronic diseases, such as pulmonary and cardiovascular disease [62]. To clean PM 2.5, Guo et al. reported an SP triboelectric negative air ion generator (MSNG) powered by TENG [63]. Powering by TENG, the voltage of carbon fiber electrodes was over $2000 \mathrm{~V}$, which can be employed to produce negative air ions in these electrodes (Figure $2 \mathrm{~h}$ ). For demonstrating the ability to clean PM 2.5, $1 \times 10^{13}$ NAIs was generated by using a palm-sized MSNG, and PM 2.5 with initial concentration of $999 \mu \mathrm{g} \mathrm{m}^{-3}$ was quickly declined to $0 \mu \mathrm{g} \mathrm{m}^{-3}$ in $80 \mathrm{~s}$ at $0.25 \mathrm{~Hz}$. Furthermore, the observable heavy smog purification processes are displayed in Figures $2(i)$ and $2(j)$, which demonstrated the high efficiency of the MSNG for air purge.

The primary pollutants of gaseous pollutants result from the original pollutants directly discharged into the atmosphere from the source, mainly including oxysulfide, oxynitride, and organic compounds [64]. For removing sulfur dioxide $\left(\mathrm{SO}_{2}\right)$ and dust, Chen et al. reported an SP air cleaning system on the basis of a rotary TENG [65]. Driven by nature wind, rotary TENG produced a high voltage about $300 \mathrm{~V}$, which was applied to power the electrochemical oxidation of $\mathrm{SO}_{2}$ without the byproducts such as ozone and NOx than conventional electrostatic precipitation. For cleaning oxynitride, Han et al. proposed an $\mathrm{SP}^{\mathrm{NO}_{\mathrm{X}}}$ absorption and degradation system on the basis of a radial-engineshaped TENG system [66]. By harvesting win energy, the $\mathrm{SP}$ system synchronously achieved the removal of $\mathrm{NO}_{\mathrm{x}}$. For cleaning formaldehyde in indoor atmosphere, Feng et al. demonstrated an SP electrostatic filter by integrating TENG with photocatalysis technology [67]. In this work, a single electrode TENG was used as power source to produce high electric field on the filtering network. Consequently, the SP electrostatic filter was demonstrated to clean formaldehyde via both electrostatic adsorption effect induced by TENG and enhanced photocatalytic effect by the photocatalyst on the SP filter networks, where the formaldehyde concentration decreased to $60 \%$ within $250 \mathrm{~min}$, and a tripled increased efficiency of formaldehyde removal was achieved.

\subsection{Self-Powered Electrochemical Synthesis System. Convert-} ing energy from environment into easily storable chemical energy such as hydrogen and formic acid has drawn public attention as the alternative technology to ensure a clean and sustainable energy supply [68]. Generally, the process of clean fuel production needs an external input power, where renewable energies such as solar, wind, geothermal, and hydro have been applied along with carbon dioxide and water to generate clean fuels [69]. Owing to the ability of harvesting ambient mechanical energy, TENG-based SP electrochemical system has been demonstrated as a promising technology for clean fuel generation [70-73]. Tang et al. firstly proposed a fully SP water splitting system for producing hydrogen [74], which integrated a rotary TENG with a water splitting unit (Figures 3(a) and 3(b)). Driven by the rotary TENG at $10 \mathrm{~Hz}$, the production ratio of hydrogen was up to $6.25 \times 10^{-3} \mathrm{~mL} \mathrm{~min}^{-1}$ in $30 \%$ (w.t.) potassium hydroxide solution. As demonstrating, obvious bubbles were observed in the electrode (Figures 3(c) and 3(d)). To improve the production speed of hydrogen, introducing a hybrid cell to increase the output power of SP water splitting system is a promising strategy [75]. Yang et al. designed an SP water splitting system on the basis of a hybrid energy cell, where the hydrogen production speed had been improved to $4 \times 10^{-4} \mathrm{~mL} \mathrm{~s}^{-1}$ [76].

As the other type of clean fuel, formic acid with characteristics of high volumetric capacity, low toxicity, and flammability under ambient condition has drawn wide attention [77]. Leung et al. presented an SP carbon dioxide reduction system that harvests energy from ocean wave for converting carbon dioxide into formic acid (Figure 3(e)) [78]. At an optimal value of discharge voltage $(2.4 \mathrm{~V})$ for each cycle, the system realized near $100 \%$ faradaic efficiency for shifting carbon dioxide into formic acid. Driven by TENG under a simulated waves with water surface area of $0.04 \mathrm{~m}^{2}$, the system generated $2.798 \mu \mathrm{M}$ of formic acid per day.

Ammonia $\left(\mathrm{NH}_{3}\right)$ acts a crucial role in food production, industrial manufacturing, and a predictable ideal energy carrier in the future [79]. In industry, Haber-Bosch process is commonly employed to produce $\mathrm{NH}_{3}$ with the existence of hydrogen and external energy supply, where the challenge of grueling conditions increases the cost of production [80]. Due to a controllable operation under mild conditions utilizing mechanical energy by TENG, the SP electrocatalytic $\mathrm{NH}_{3}$ synthesis system based on TENG provides a promising candidate for the conversion of $\mathrm{N}_{2}$ to $\mathrm{NH}_{3}$. Gao et al. reported an SP sustainable metal-free $\mathrm{NH}_{3}$ production system based on a multilayer asway TENG by $3 \mathrm{D}$ printing technology, which can efficiency convert $\mathrm{N}_{2}$ into $\mathrm{NH}_{3}$ [81]. The maximum power density of $6.7 \mathrm{~W} \mathrm{~m}^{-2}$ was realized by the printed TENG, which was used to drive the production of $\mathrm{NH}_{3}$. By using the carbon materials from melamine sponge as the metal-free electrocatalyst, the assembled $\mathrm{SP} \mathrm{NH}_{3}$ production system can reach $\mathrm{NH}_{3}$ yield of $36.41 \mu \mathrm{g} \mathrm{h}^{-1} \mathrm{mg}^{-1}$ cat. By introducing a high-output dual-TENG configuration, Han et al. constructed an SP electrocatalytic $\mathrm{NH}_{3}$ synthesis system to simultaneously achieve nitrogen fixation and electrocatalytic reduction with air as the $\mathrm{N}_{2}$ source [82]. The electrocatalytic $\mathrm{NH}_{3}$ synthesis system mainly consisted of three parts, including TENG-1, a needle-plate, and TENG-2. Specifically, TENG-1 was used to produce high voltage, a needle-plate was utilized to fix nitrogen and produce $\mathrm{NO}_{\mathrm{X}}$ which then flowed into a water receptacle to produce $\mathrm{NO}_{3}{ }^{-}$and $\mathrm{NO}_{2}{ }^{-}$, and TENG-2 was employed to generate pulsed DC which drove 

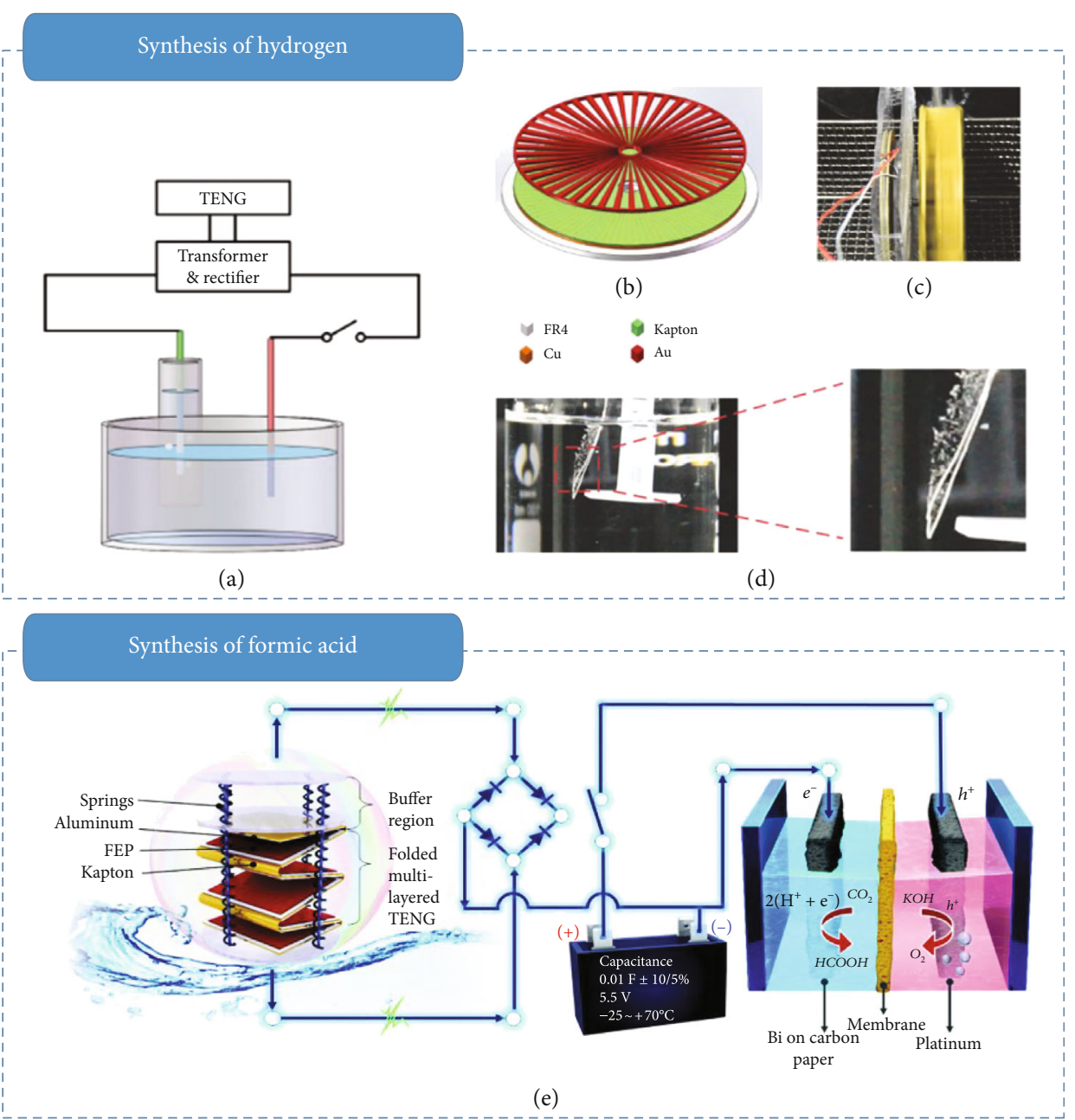

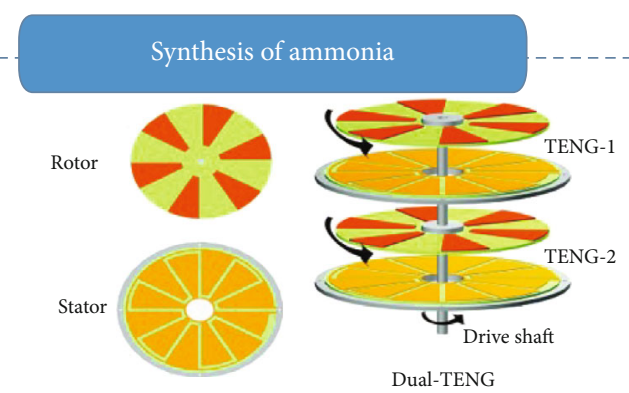

(f)

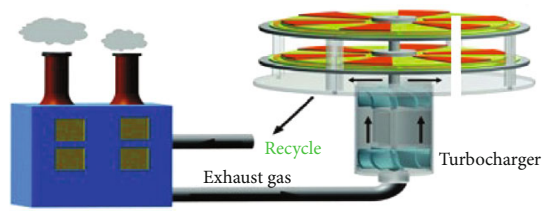

(h)

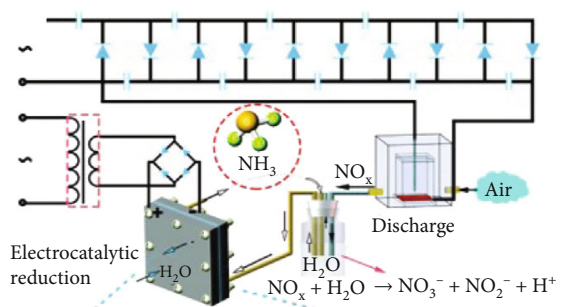

(g)

FIGURE 3: Self-powered electrochemical synthesis: (a) sketch map of the SP water splitting system; (b) structure of the disk TENG; (c) the SP water splitting system driven by a miniature water turbine; (d) images of the system with bubble producing [74]; (e) sketch map of the SP electrochemical system for $\mathrm{CO}_{2}$ reduction powered by TENG for harvesting ocean-wave energy [70]; (f) sketch map of the designed TENG; (g) reaction of SP ammonia synthesis system; (h) sketch map of the SP structure driven by exhaust gas; ( $i, j$ ) concentration of $\mathrm{NH}_{3}$ in the cathode compartment at different times (i) and the volume of residual solution and the yield of $\mathrm{NH}_{3}(\mathrm{j})$ by the SP system after ten hours [82]. 
the reaction for ammonia production (Figures $3(\mathrm{f})$ and $3(\mathrm{~g})$ ). Driven by the gas flow, a high voltage of $7 \mathrm{kV}$ supplied by TENG-1 and the output performance of $3.1 \mathrm{~V}$ and $8.9 \mathrm{~mA}$ for the cell reaction supplied by TENG-2 were achieved, respectively (Figure $3 \mathrm{~h}$ ). By employing the SP electrocatalytic $\mathrm{NH}_{3}$ synthesis system based on above dual-TENG, $2.4 \mu \mathrm{g} \mathrm{h}{ }^{-1}$ of $\mathrm{NH}_{3}$ was successfully synthesized (Figure 3(i) and 3(j)).

Besides the abovementioned works, some other SPESs such as SP electrochemical oxidation system and SP electrodeposition system were also developed to electrochemical synthesis [83-86]. Zheng et al. reported an SP electrochemical oxidation system on the basis of designed cross-linked TENG for synthesizing polyaniline [85]. As for the crosslinked TENG, the high capability of $69.9 \mu \mathrm{A}$ and $845.6 \mathrm{~V}$ was achieved. By harvesting vibration energy, the SP electrochemical oxidation system was utilized as electricity source for converting aromatic amines to polyaniline. Wang et al. established a self-powered electrodeposition system for polypyrrole synthesis, where the polypyrrole as the electrode material of TENG was produced by TENG [86].

3.3. Self-Powered Electrochemical Sensor System. Based on TENG, lots of SP electrochemical sensors have been proposed to detect chemical substances. According to the mechanism of sensing, SP electrochemical sensor can be classified into two types including SP electrochemical passive sensor and SP electrochemical active sensor [47, 87].

SP electrochemical passive sensor is that the conventional sensors are driven by TENG for collecting mechanical energy from environment. Zhang et al. proposed an SP glucose biosensor based on contact-separation type TENG combined with lithium-ion battery [88]. The flexible TENG fabricated by a patterned polydimethylsiloxane (PDMS) film can extract energy from the motion of clapping; thus, the battery with an increased charging voltage from $400 \mathrm{mV}$ to $800 \mathrm{mV}$ was achieved after more than two hours, which was successfully demonstrated to power a glucose biosensor. Aiming to voluntarily supervise the water quality, Bai et al. reported an in situ SP sensing system that can convert the water wave energy to electricity based on tandem disk TENG [89]. Owing to the radial grating disk structure with swinging mass blocks, the tandem disk TENG driven by water waves can realize a conversion from low-frequency water wave motions into high-frequency output; thus, an average power density of $7.3 \mathrm{~W} \mathrm{~m}^{-3}$ was achieved. Through rectification and energy storage, the electricity produced by TENG can be utilized to drive electronics for monitoring the water quality.

As for the SP electrochemical active sensor, a TENG is designed to actively generate electrical signal for responding the stimulation of chemical molecules or environmental factors such as ethanol, phenol, catechin, and $\mathrm{pH}$, where the output performance of TENG and the target shows a linear relationship [51, 90-92]. Zhang et al. demonstrated SP sensors based on the TENGs fabricated by polyamide (PA) film and polytetrafluoroethylene (PTFE) film for detecting liquid/gaseous ethanol [90]. The TENG was mainly composed of two plates, where a layer of PA or PTFE film was pasted on a copper foil as back electrode, and a nanopore modified Al foil was used as the triboelectric electrode. Due to the degrees of wettability of the PA and PTFE films to ethanol, the output voltage of the SP sensor logarithmically declined with the concentration of ethanol solutions increasing from $20 \%$ to $80 \%$, as well as the output signal decreased with the increase of ethanol gas concentration ranging from $40 \%$ to $80 \%$. Lin et al. proposed a contact-separation-type TENG as an SP nanosensor toward catechin detection [91]. For this TENG, PTFE film and a layer of $\mathrm{TiO}_{2}$ nanomaterial (nanowire and nanosheet) array were used as a pair of triboelectric materials. Because of the strong interaction between Ti atoms of $\mathrm{TiO}_{2}$ nanomaterial and enediol group of catechin, a high sensitivity (detection limit of $5 \mu \mathrm{M}$ ) and a linear range from $10 \mathrm{M}$ to $0.5 \mathrm{mM}$ of the SP nanosensor were achieved, demonstrating great potential for the determination of catechin concentrations in real samples. Wu et al. reported an SP triboelectric sensor based on the sliding type TENG for detecting $\mathrm{pH}$ value from a periodic contact/separation motion [92]. A fork-finger structure was designed for SP triboelectric sensor, which mainly consisted of fluorinated ethylene-propylene (FEP) film and metal electrodes. The reciprocating motion between SP triboelectric sensor and buffer solution resulted in charge transfer between the adjacent $\mathrm{Cu}$ bottom electrodes, generating AC voltage in the external circuit. The output voltage of the SP triboelectric sensor enhanced with increased $\mathrm{pH}$ value due to the increased ion concentration. Therefore, the $\mathrm{pH}$ value of buffer solution can be actively monitored in real-time by reading the output voltage. $\mathrm{Li}$ et al. proposed an SP active sensor to $\mathrm{Hg}^{2+}$ ions monitoring based on TENG where 3-mercaptopropionic acid-modified gold nanoparticles was used as recognition element [93]. In this system, a contact-separation-type TENG was employed, which shown a layered structure based on two plates (Figure 4(a)). The output performance of TENG and the concentration of $\mathrm{Hg}^{2+}$ ions displayed a linear relationship because the chemical potential difference between the different triboelectric layers defined the triboelectrification effects. As shown in the inset of Figure 4(b), the output performance of the TENG declined with improving the concentration of $\mathrm{Hg}^{2+}$ ions, where the short-circuit current ratio was proportional to the concentration of $\mathrm{Hg}^{2+}$ ions in the range of $100 \mathrm{nM}-5000 \mathrm{nM}$. Due to the high selectivity of 3-mercaptopropionic acid toward $\mathrm{Hg}^{2+}$ ions, $\mathrm{Hg}^{2+}$ ion can be specifically detected by the proposed sensing system (Figure 4(b)). Jie et al. demonstrated an SP triboelectric sensor for monitoring dopamine in the alkaline condition on the bases of TENG, which was composed of PTFE with nanoparticle arrays and an Al film (Figures 4(c) and 4(d)) [94]. Because the nano-stick PTFE exhibits a strong interaction of dopamine, the output performance of the TENG was inversely proportional to the concentration of dopamine in the range of $10 \mu \mathrm{M}-1$ $000 \mu \mathrm{M}$ (Figure 4(e)). In this work, a detection limit of dopamine was $0.5 \mu \mathrm{M}$, which suggested an effective means of SP electrochemical sensor for dopamine detection. Wen et al. introduced an SP gas sensor on the basis of a blowdriven TENG, where a rotary TENG was applied as shown in Figure 4(f) [95]. The illustration of the SP breath analyzer is shown in Figure 4(g). Driven by mouth blowing, 


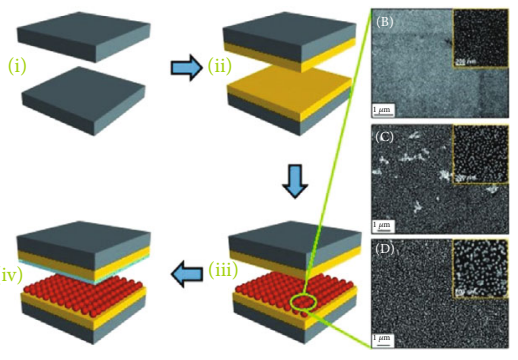

(a)

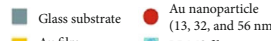

Au film PDMS film

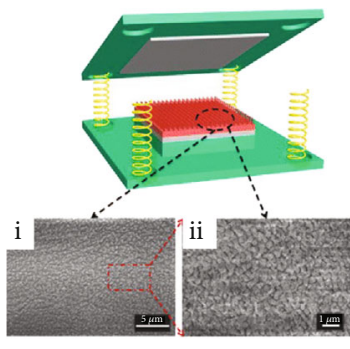

(c)

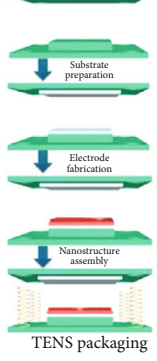

(d)

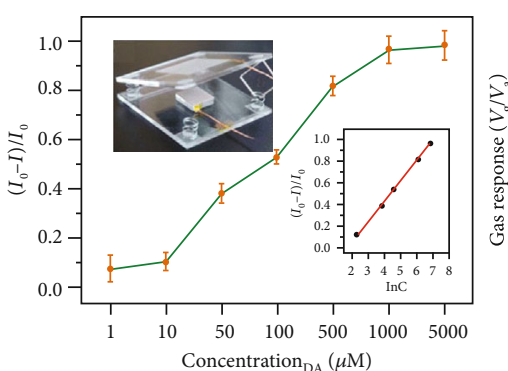

(e)

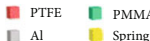
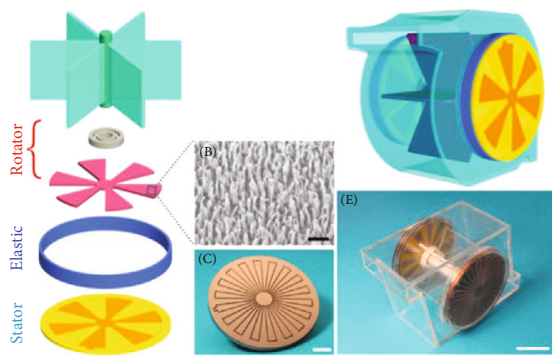

(f)

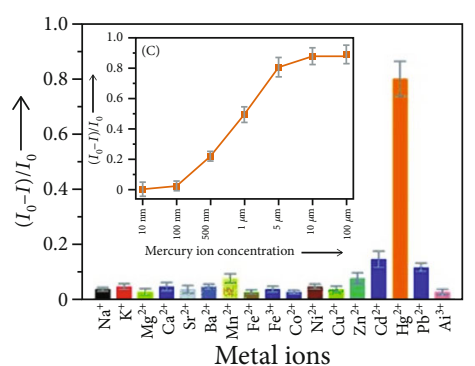

(b)

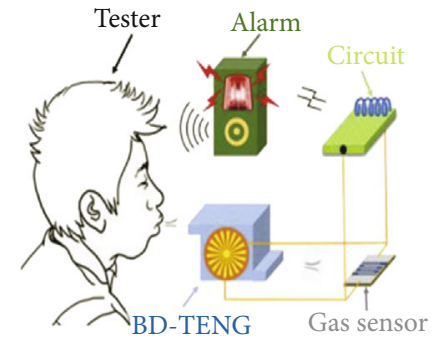

(g)

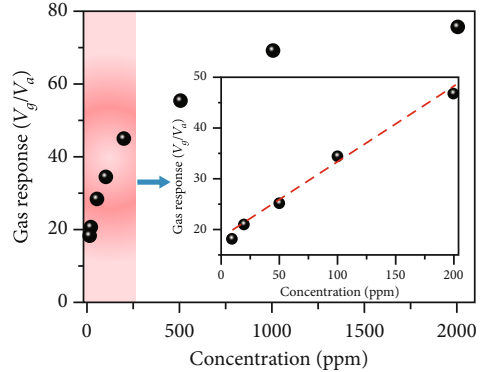

(h)

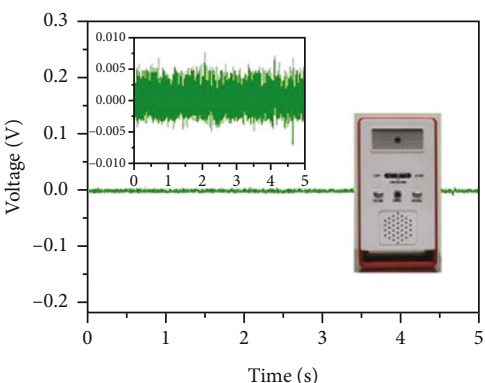

(j)

Acrylic Elastic
Bearing Cu electrode
FEP

(i)

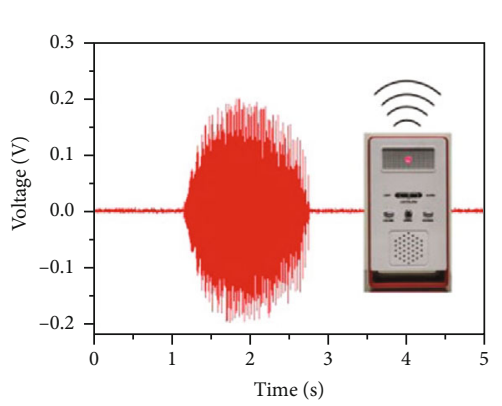

FIGURE 4: Self-powered electrochemical sensor: (a) fabrication process and the structure of the TENG; (b) selectivity of the SP electrochemical sensor for testing $\mathrm{Hg}^{2+}$ ions. Inset shows the sensitivity of $\mathrm{Hg}^{2+}$ ions detection [93]. (c, d) Sketch map and the fabrication process of the TENG; (e) sensitivity of the SP active sensor in supervising dopamine. Insets show the structure of designed TENG and proportional relation between output performance of TENG and the concentration of dopamine [94]. (f) Sketch map of the blow-driven TENG; (g) a schematic illustration the blow-driven TENG using as an SP breath analyzer; (h) response curve of the measured gas in terms of output voltage. Inset is the schematic illustration of a self-power breath analyzer; $(i, j)$ voltage signals of the system with warning alarm by a person before and after drinking alcohol [95].

the output voltage of the sensor was only proportional to the concentration of alcohol in the airflow (Figure 4(h)). On the basis of blow-driven TENG, the active alcohol breath analyzer exhibited a high detection gas response of $\sim 34$ under an optimized condition. Additionally, when the blow-driven TENG was blew by a tester without drinking alcohol, the voltage drop across the sensor was almost zero due to a low sensor resistance (Figure 4(i)). While the air-flow of a tester that had been drank across the sensor, an enhanced voltage would be generated and thus could trigger the warning system (Figure $4(\mathrm{j})$ ), which was caused by the dramatically increased resistance of the sensor in the breathed-out alcohol vapor.

3.4. Self-Powered Electrochromic System. Electrochromic devices can reversibly change their optical properties by the electrochemical redox reaction under an external electric field [96]. Applied TENG as the electricity source to provide a constant voltage, SP electrochromic device has been realized to replace batteries, which provides a promising sustainable power solution $[97,98]$. 

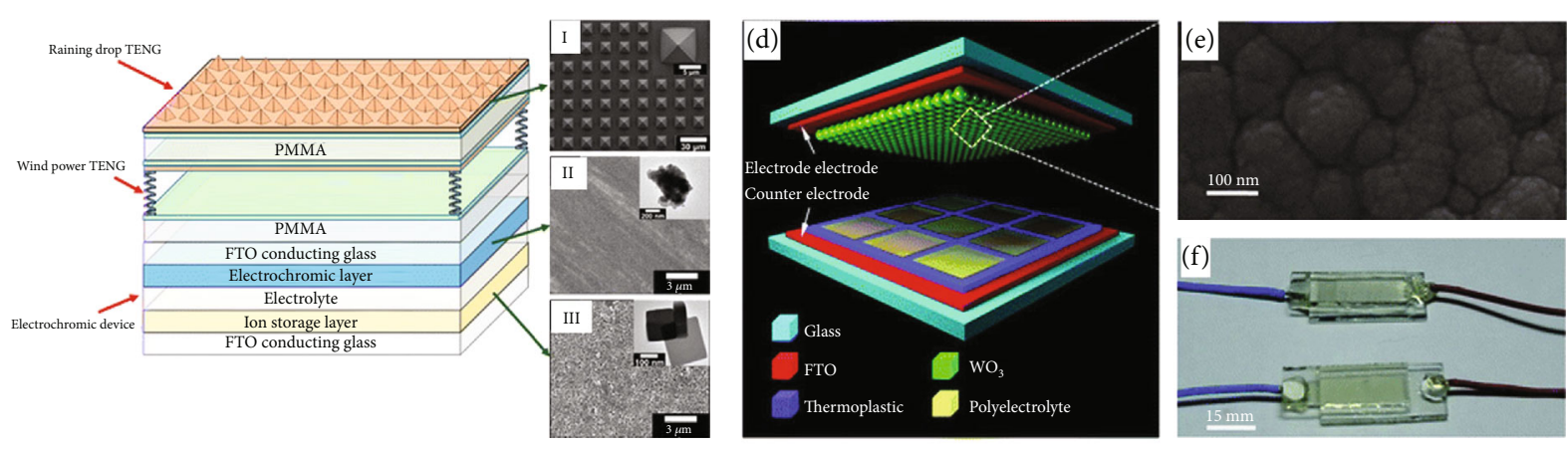

(a)

ITO/PET film

PDMS film

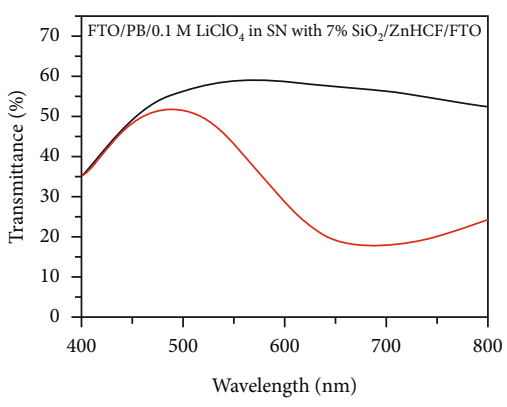

(b)

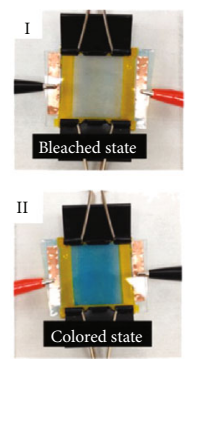

(c)

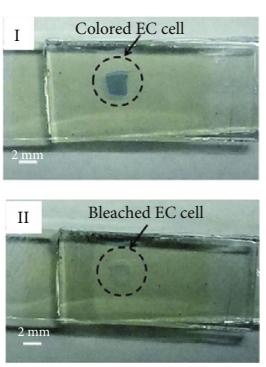

(g)

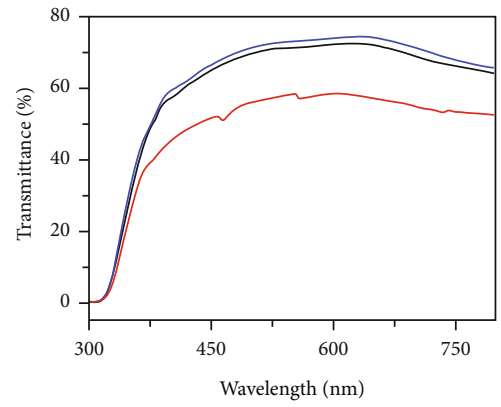

(h)

Bleached state

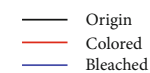

FIGURE 5: Self-powered electrochromic reaction: (a) schematic diagram of the SP smart window integrated with a raindrop-TENG, a windpowered-TENG, and an electrochromic device from the top to the bottom; inserts are the SEM images of (I) PDMS film, (II) PB film, and the (III) ZnHCF film; (b) transmittance spectra and (c) photographs of the SP smart window system in bleached and colored states [99]; (d) the schematic structure of the $\mathrm{WO}_{3}$-based SP electrochromic device; (e) SEM image of the $\mathrm{WO}_{3}$ film; (f) the photographs of the EC WO $\mathrm{W}_{3}$-based SP electrochromic device; (g) color changing images of the $\mathrm{WO}_{3}$-based SP electrochromic device; (h) transmittance spectra of the $\mathrm{WO}_{3}$-based SP electrochromic device [100].

Driven by a dual-mode TENG for harvesting wind and raindrop energy, Yeh et al. realized an SP smart window system [99]. The dual-mode TENG involved a single-electrode TENG on the top of the SP smart window for harvesting the energy from raindrop motions, and a contact-mode TENG assembled by elastic springs below the abovementioned single-electrode-mode TENG for collecting energy from wind energy. Both of the two TENGs consisted of a PDMS thin film adhered to a conducting substrate that were adhered to the electrochromic device with a substrate to fabricate an SP system (Figure 5(a)). The electrochromic device mainly consisted of Prussian blue (PB) nanoparticles and zinc hexacyanoferrate ( $\mathrm{ZnHCF}$ ) nanocubes as the electrochromic material and the ion storage layer. By photolithography and a template molding process, the PDMS film has a micropatterned pyramid array structure for enhancing the hydrophobic property and effect contact area of the surface. Driven by the dual-TENG, the transmittance of the electrochromic exhibited reversible variations. For gaining a more intuitive view of the change of the optical property, the transmittance of the electrochromic device was measured from 400 to $800 \mathrm{~nm}$ both before and after the coloring process. As shown in Figure 5(b), the transmittance declined in the full range during the coloring process, and the highest variation was realized at $695 \mathrm{~nm}$, while the transmittance declined from $53.5 \%$ to $20.9 \%$. These transmittance changes can be observed by visualization, where the color of the electrochromic device changed from transparent in bleached state to deep blue in colored state (Figure 5(c)). In this work, $32.4 \%$ of the maximum transmittance change was achieved at $695 \mathrm{~nm}$, which closed to the value of $32.6 \%$ that powered by a conventional electrochemical power source.

Yang et al. reported a $\mathrm{WO}_{3}$-based electrochromic device integrating with TENG to fabricate an SP electrochromic device [100]. The SP electrochromic device had a multilayered structure, which is displayed in Figure 5(d). Commercial glass was used as the substrate, on which there was a layer of FTO thin films acted as electrodes. The sheet resistance and transmittance of FTO film were $35-45 \Omega$ per sq. and $80 \%$, respectively. Between the two electrodes, there were an array of cells and a layer of $\mathrm{WO}_{3}$ film (Figure 5(e)), and the distance between the two electrodes was around $20 \mathrm{~mm}$. The cells were filled with polyelectrolyte, and the $\mathrm{WO}_{3}$ film was about $250 \mathrm{~nm}$ which was densely packed nanoparticles. As shown in Figure 5(f), the fully packaged SP electrochromic device still had a transmittance of more 


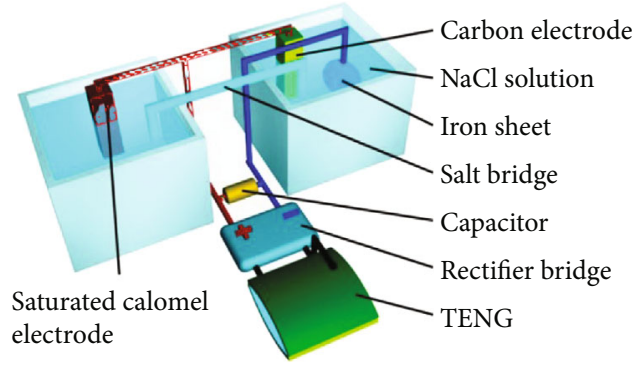

(a)
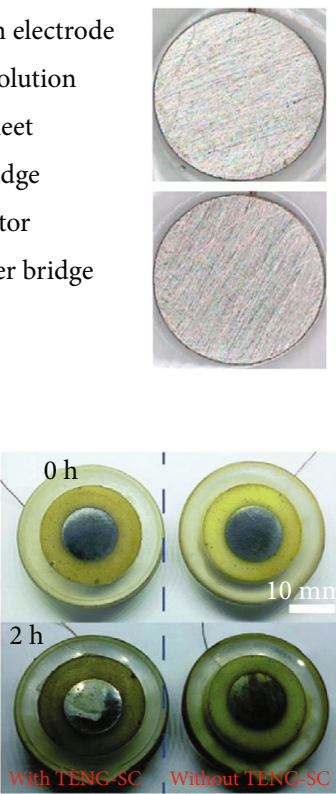

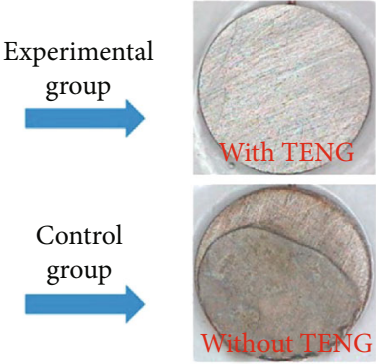

(b)

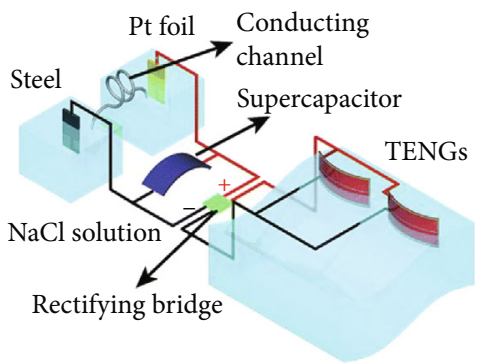

(c)

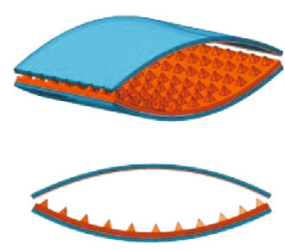

(e)

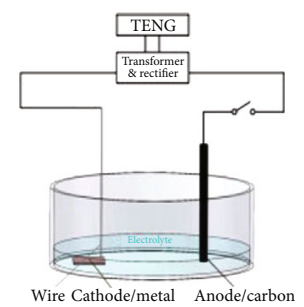

(f)

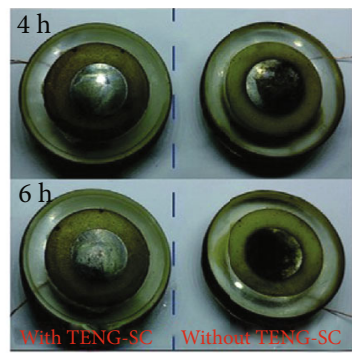

(d)

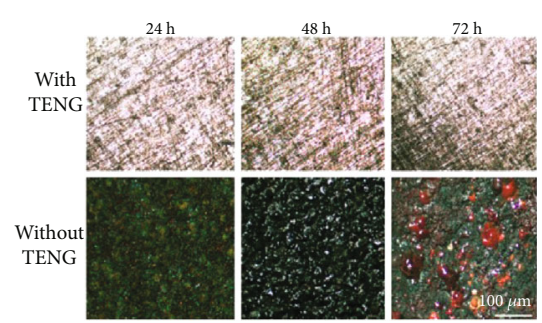

(g)

$$
\begin{aligned}
& \text { PET } \\
& \text { ITO } \\
& \text { PDMS }
\end{aligned}
$$

FiguRE 6: Self-powered anticorrosion system: (a) sketch map of the SP anticorrosion system; (b) iron sheets before and after soaked in the solution for $2 \mathrm{~h} \mathrm{[112];} \mathrm{(c)} \mathrm{sketch} \mathrm{map} \mathrm{of} \mathrm{the} \mathrm{SP} \mathrm{anticorrosion} \mathrm{system} \mathrm{powered} \mathrm{by} \mathrm{TENG;} \mathrm{(d)} \mathrm{the} \mathrm{contrast} \mathrm{images} \mathrm{of} \mathrm{the} \mathrm{surface}$ morphology of the samples after immersing in sodium chloride solution driven by TENG or not [113]; (e) scheme of the designed TENG; (f) schematic diagram of the metal surface cathodic protection system driven by TENG; (g) micrographs of the rusted specimens after accelerated corrosion [110].

than $70 \%$. As shown in Figure 5(g), powered by TENG, the transmittance of electrochromic cell was drop via coloring process (Figure 5(g), (I)). When the connection of reversible switch was reversed, the electrochromic cell returned to transparency (Figure 5(g), (II)). Compared with the SP electrochromic device, the transmittance of the device greatly dropped, and a relatively stable difference of as much as $17 \%$ was maintained for wavelength that ranges from $450 \mathrm{~nm}$ to $650 \mathrm{~nm}$ (Figure 5(h)), indicating the sensitivity and the applicable to a broad range of light wavelength.

To effectively harvest acoustic energy, Qiu et al. integrated a sandwich-like structured TENG with electrochromic device for reversible color changing [101]. The TENG consisted of three layers including $\mathrm{Cu}$ foam, polyvinylidene fluoride (PVDF) nanofiber, and a nylon fabric. There was a thick spacer layer between the nylon fabric and PVDF nanofibers to produce more space vibration for the membrane. Driven by sound, high outputs of $25.01 \mathrm{~mA} \mathrm{~m}^{-2}$ and $20.91 \mu \mathrm{C} \mathrm{s}^{-1}$ were achieved for the designed TENG. The high performance of TENG enabled it to power an electrochromic device. In this system, reversible switches controlled the oxidation and reduction process and thus controlled the color change. Driven by the TENG, the color of electrochromic film changed between the transparent white and dark blue, which were controlled by the switching dote.

3.5. Self-Powered Anticorrosion System. Material corrosion is a long-standing challenge in many engineering applications [102]. The cathodic protection is one of the most durable methods to protect the materials from corrosion, which involves sacrificial anode cathodic protection system (SACPS) and impressed current cathodic protection system (ICCPS) [103-105]. Different from SACPS, the ICCPS can protect the steel by cathodic current from a direct current source without any sacrifice of electrode materials [106]. 
However, the requirement of external electric energy supply limits its practical application. For solving this issue, many works have been focused on the SP anticorrosion system by integrating TENG with chemical anticorrosion protection [107-111].

Wang et al. realized an SP anticorrosion system for iron sheet driven by a high performance TENG [112]. Utilizing the fabricated nanostructures and prior-charge injection method, the charge densities were improved by $48 \%$ and $53 \%$, respectively. The schematic image of SP anticorrosion system is depicted in Figure 6(a). Aiming to study the effect of SP anticorrosion system, the ion steel was soaked into $3.5 \% \mathrm{NaCl}$ solution for $2 \mathrm{~h}$, and the corresponding surface morphology is displayed in Figure 6(b). As displayed, almost no change of the iron sheet was observed by using TENG, while a thin rust film was produced without TENG. This result indicated a good anticorrosion property of the SP system, which exhibited a good prospect to protect materials from rusting with low energy cost.

Considering the metal corrosion is more likely to happen under ocean environmental conditions, Li et al. developed an SP system on the basis of networking TENG and supercapacitor to convert water wave to electricity for metal anticorrosion [113]. To obtain a stable and continuous output, the flexible TENG integrated with a flexible double-layer supercapacitor for harvesting the wave energy and then storing the energy in the supercapacitor. The structure and working mechanism of the SP system displayed in Figure 6(c). Driven by the water wave in the condition of $0.2 \mathrm{~m} \mathrm{~s}^{-1}$ and $1 \mathrm{~Hz}$, the protected steels injected into electrons stemmed from TENG, leading to a cathodic polarization. In this work, a declined potential of steel electrode from $-0.35 \mathrm{~V}$ to $-0.6 \mathrm{~V}$ indicated that the steel become more stable in the protection of designed SP system. Immersing in the solution of sodium chloride with concentration of $0.5 \mathrm{M}$, there was a thin layer of rust on the steel without the protection of SP system, while there were a few corrosion pits on the steel when the SP system was working (Figure 6(d)). These results demonstrated that the SP system significantly decreased the corrosion rate, which could be adopted in marine corrosion.

Zhu et al. designed an SP cathodic protection system based on a flexible TENG, which can harvest energy from natural rain drops and wind to drive the cathodic protection process [110]. The contact-separation-type TENG was mainly composed of PDMS film and ITO, which were acted as a pair of triboelectric layers (Figure 6(e)). The schematic diagram of the SP metal surface cathodic protection system is shown in Figure 6(f). Under stimulations at a frequency of $1 \mathrm{~Hz}$, the output current of the TENG was over $130 \mu \mathrm{A}$, and the voltage reached about $500 \mathrm{~V}$. In this SP cathodic protection system, the protected metal that was immersed into the electrolyte was acted as the cathode, while a carbon rod was used as the anode. The comparison results of the rested specimens with and without SP cathodic protection system are displayed in Figure 6(g). For the specimens protected with SP cathodic protection system, several gray distributing areas appeared on the surface of specimens. These areas enlarge with the increase of corrosion, but no apparent corrosion was observed on these samples. As for the specimen without SP cathodic protection system powered by TENG, numerous corrosion pits were found on the three surfaces of specimens, and more big pits arose on the sample that was corroded in simulated electrolyte for $72 \mathrm{~h}$.

\section{Summary and Perspective}

Electrochemistry has brought earth-shaking changes to our life, and it has become a technology for production and research in many fields. In order to break the limitation of external powers source for electrochemical operation, integrating electrochemical system with TENG to form SPES is the most promising strategies. According to the latest achievements, SPESs can be summarized into five major fields including pollutants treatment, electrochemical synthesis, electrochemical sensor, electrochromic reaction, and metal anticorrosion, respectively. Although researches into SPES have realized remarkable progresses, the following issues should be addressed for promoting further development of this field:

(1) High Output Power and Durability of TENG. Output power and durability of TENG are two key points to realize high performance of SPES. To improve the output power of TENG, further improving the surface charge density and integrating existing methods in a large scale are suggested. To enhance the durability of TENG, introducing interface liquid lubrication [114] and developing materials with the most robust mechanical durability and stability might be the promising strategies

(2) Power Circuit Management of TENG. TENG has the characteristics of high voltage output and low current output, while the performance of electrochemical process exhibits positive correlation with current density and negative influence such as passive electrode effect and secondary reaction induced by high potential. To achieve a high performance of SPES, the power circuit management of TENG is highly desired to match the corresponding electrochemical process

(3) Reducing electrode passive is crucial to prolong the lifetime of electrodes, improve the electrochemical efficiency, and ensure a stable activity. TENG with a pulse output signal has been reported to reduce the electrode passive effect; however, the phase superposition of TENG caused by multiple parallel electrodes makes it hard to realize a full-waveform-pulse-current. Therefore, rational design of the structure of TENG, such as adjusting the rotation center angle ratio between each rotator and stator to obtain a full-waveform-pulse-current, will be a promising strategy to optimize the processes of SPES. It has been reported that alternating current exhibits many virtues such as lower energy consumption, improved mass transfer characteristic, and delayed electrode passivation compared to direct current in the electrochemical system [115]; thus, utilizing the alternating 
current of TENG to build SP electrode system is the other method to reduce the electrode passive and improve the electrochemical performance

(4) The electrode plays a key role in the electrochemical reaction. Therefore, the electrode materials are the most key factors to determine the properties of electrochemical reaction. Novel electrode materials such as nanoscale electrode materials and metal-organic framework materials with merits of high conductivity, high specific surface areas, high activity, and cycle stability should be prepared to further enhance the performance of SPES

(5) New Application of SPES. With the improvement performance of TENG, it can be utilized as electricity source to power some new electrochemical reaction such as electrocoagulation for removal of oil in water, electrodialysis for desalination and water reuse, and electrophoresis for the separation of protein to build SPES for overcoming the problem of external power supply. Besides traditional electrochemistry based on electrolytic cell that needs high current density, the interrelation of electrical and chemical effect can also be realized by high voltage electrostatic discharge. By utilizing TENG, a high voltage electrostatic is actually quite easy to achieve. Therefore, integrating TENG with electrochemical system to build SP discharge electrochemical system for removing pollutants such as PM [63] provides a new horizon for its application

\section{Conflicts of Interest}

The authors declare no conflict of interest.

\section{Authors' Contributions}

Linglin Zhou and Di Liu contributed equally to this work.

\section{Acknowledgments}

This review was supported by the National Key R\&D Project from Ministry of Science and Technology (2016YFA0202701), the National Natural Science Foundation of China (Grant Nos. 61774016, 21773009, 51432005, 5151101243, and $51561145021)$, and the Beijing Municipal Science \& Technology Commission (Z171100000317001, Z171100002017017, and Y3993113DF).

\section{References}

[1] X. L. Ma, J. C. Liu, H. Xiao, and J. Li, "Surface single-cluster catalyst for $\mathrm{N}_{2}$-to- $\mathrm{NH}_{3}$ thermal conversion," Journal of the American Chemical Society, vol. 140, no. 1, pp. 46-49, 2018.

[2] J. Li, E. Kim, K. M. Gray et al., "Mediated electrochemistry to mimic biology's oxidative assembly of functional matrices," Advanced Functional Materials, vol. 30, no. 30, article 2001776, 2020.

[3] J. Xie and Y. Wang, "Recent development of CO2 electrochemistry from $\mathrm{Li}-\mathrm{CO} 2$ batteries to $\mathrm{Zn}-\mathrm{CO} 2$ batteries,"
Accounts of Chemical Research, vol. 52, no. 6, pp. 17211729, 2019.

[4] C. A. Martínez-Huitle and E. Brillas, "Decontamination of wastewaters containing synthetic organic dyes by electrochemical methods: a general review," Applied Catalysis B: Environmental, vol. 87, no. 3-4, pp. 105-145, 2009.

[5] A. Morozan and F. Jaouen, "Metal organic frameworks for electrochemical applications," Energy \& Environmental Science, vol. 5, no. 11, article 9269, 2012.

[6] Z. L. Wang and J. Song, "Piezoelectric nanogenerators based on zinc oxide nanowire arrays," Science, vol. 312 , no. 5771, pp. 242-246, 2006.

[7] Z. L. Wang, "Energy harvesting for self-powered nanosystems," Nano Research, vol. 1, no. 1, pp. 1-8, 2008.

[8] Z. L. Wang, "Towards self-powered nanosystems: from nanogenerators to nanopiezotronics," Advanced Functional Materials, vol. 18, no. 22, pp. 3553-3567, 2008.

[9] Z. L. Wang, "Self-powered nanotech," Scientific American, vol. 298, no. 1, pp. 82-87, 2008.

[10] S. Xu, B. J. Hansen, and Z. L. Wang, "Piezoelectric-nanowireenabled power source for driving wireless microelectronics," Nature Communications, vol. 1, no. 1, p. 93, 2010.

[11] P. G.-J. M. El-hami, N. M. White, M. Hill et al., "Design and fabrication of a new vibration-based electromechanical power generator," Sensors and Actuators A: Physical, vol. 92, no. 1-3, pp. 335-342, 2001.

[12] F.-R. Fan, Z.-Q. Tian, and Z. Lin Wang, "Flexible triboelectric generator,” Nano Energy, vol. 1, no. 2, pp. 328-334, 2012.

[13] Z. L. Wang, "Triboelectric nanogenerators as new energy technology and self-powered sensors-Principles, problems and perspectives," Faraday Discussions, vol. 176, pp. 447$458,2014$.

[14] L. Huang, S. Lin, Z. Xu et al., "Fiber-based energy conversion devices for human-body energy harvesting," Advanced Materials, vol. 32, article 1902034, 2019.

[15] H. Guo, X. He, J. Zhong et al., "A nanogenerator for harvesting airflow energy and light energy," Journal of Materials Chemistry A, vol. 2, no. 7, pp. 2079-2087, 2014.

[16] Z. L. Wang, J. Chen, and L. Lin, "Progress in triboelectric nanogenerators as a new energy technology and selfpowered sensors," Energy \& Environmental Science, vol. 8, no. 8, pp. 2250-2282, 2015.

[17] J. Wang, S. Li, F. Yi et al., "Sustainably powering wearable electronics solely by biomechanical energy," Nature Communications, vol. 7, no. 1, article 12744, 2016.

[18] K. D. X. Peng, C. Ye, Y. Jiang et al., “A breathable, biodegradable, antibacterial, and self-powered electronic skin based on all-nanofiber triboelectric nanogenerators," Science Advance, vol. 6, article eaba9624, 2020.

[19] Y.Zou, P. Tan, B. Shi et al., "A bionic stretchable nanogenerator for underwater sensing and energy harvesting," Nature Communications, vol. 10, no. 1, article 2695, 2019.

[20] X. P. H. Guo, J. Chen, Y. Meng et al., "A highly sensitive, selfpowered triboelectric auditory sensor for social robotics and hearing aids," Science Robot, vol. 3, no. 20, article eaat2516, 2018.

[21] H. Wang, L. Xu, Y. Bai, and Z. L. Wang, "Pumping up the charge density of a triboelectric nanogenerator by chargeshuttling," Nature Communications, vol. 11, no. 1, article 4203, 2020. 
[22] Z. L. Wang, "Catch wave power in floating nets," Nature, vol. 542, no. 7640, pp. 159-160, 2017.

[23] D. E. McCoy, T. Feo, T. A. Harvey, and R. O. Prum, "Structural absorption by barbule microstructures of super black bird of paradise feathers," Nature Communications, vol. 9, no. 1, p. $1,2018$.

[24] Z. L. Wang and A. C. Wang, "On the origin of contactelectrification," Materials Today, vol. 30, pp. 34-51, 2019.

[25] M. Kunitski, N. Eicke, P. Huber et al., "Double-slit photoelectron interference in strong-field ionization of the neon dimer," Nature Communications, vol. 10, no. 1, p. 1, 2019.

[26] A. Bhardwaj, J. Kaur, M. Wuest, and F. Wuest, "In situ click chemistry generation of cyclooxygenase-2 inhibitors," Nature Communications, vol. 8, no. 1, p. 1, 2017.

[27] W. Liu, Z. Wang, G. Wang et al., "Integrated charge excitation triboelectric nanogenerator," Nature Communications, vol. 10, no. 1, article 1426, 2019.

[28] L. Cheng, Q. Xu, Y. Zheng, X. Jia, and Y. Qin, "A selfimproving triboelectric nanogenerator with improved charge density and increased charge accumulation speed," Nature Communications, vol. 9, no. 1, article 3773, 2018.

[29] Y. Liu, W. Liu, Z. Wang et al., "Quantifying contact status and the air-breakdown model of charge-excitation triboelectric nanogenerators to maximize charge density," Nature Communications, vol. 11, no. 1, article 1599, 2020.

[30] W. He, W. Liu, J. Chen et al., "Boosting output performance of sliding mode triboelectric nanogenerator by charge spaceaccumulation effect," Nature Communications, vol. 11, no. 1, article 4277, 2020.

[31] S. Niu, X. Wang, F. Yi, Y. S. Zhou, and Z. L. Wang, "A universal self-charging system driven by random biomechanical energy for sustainable operation of mobile electronics," Nature Communications, vol. 6, no. 1, article 8975, 2015.

[32] W. Liu, Z. Wang, G. Wang et al., "Switched-capacitor-convertors based on fractal design for output power management of triboelectric nanogenerator," Nature Communications, vol. 11, no. 1, article 1883, 2020.

[33] X. Pu, M. Liu, L. Li et al., "Efficient charging of Li-Ion batteries with pulsed output current of triboelectric nanogenerators," Advanced Science, vol. 3, no. 1, article 1500255, 2016.

[34] G. Zhu, Y. S. Zhou, P. Bai et al., "A shape-adaptive thin-filmbased approach for $50 \%$ high-efficiency energy generation through micro-grating sliding electrification," Advanced Materials, vol. 26, no. 23, pp. 3788-3796, 2014.

[35] L. Zhou, D. Liu, J. Wang, and Z. L. Wang, "Triboelectric nanogenerators: fundamental physics and potential applications," Friction, vol. 8, no. 3, pp. 481-506, 2020.

[36] C. Wu, A. C. Wang, W. Ding, H. Guo, and Z. L. Wang, "Triboelectric nanogenerator: a foundation of the energy for the new era," Advanced Energy Materials, vol. 9, no. 1, article 1802906, 2019.

[37] S. Wang, L. Lin, and Z. L. Wang, "Nanoscale triboelectriceffect-enabled energy conversion for sustainably powering portable electronics," Nano Letters, vol. 12, no. 12, pp. 6339-6346, 2012.

[38] S. Wang, L. Lin, Y. Xie, Q. Jing, S. Niu, and Z. L. Wang, "Sliding-triboelectric nanogenerators based on in-plane chargeseparation mechanism," Nano Letters, vol. 13, no. 5, pp. 2226-2233, 2013.
[39] S. Niu, Y. Liu, S. Wang et al., "Theoretical investigation and structural optimization of single-electrode triboelectric nanogenerators," Advanced Functional Materials, vol. 24, no. 22, pp. 3332-3340, 2014.

[40] Y. Yang, Y. S. Zhou, H. Zhang, Y. Liu, S. Lee, and Z. L. Wang, "A single-electrode based triboelectric nanogenerator as selfpowered tracking system," Advanced Materials, vol. 25, no. 45, pp. 6594-6601, 2013.

[41] S. Wang, Y. Xie, S. Niu, L. Lin, and Z. L. Wang, "Freestanding triboelectric-layer-based nanogenerators for harvesting energy from a moving object or human motion in contact and non-contact modes," Advanced Materials, vol. 26, no. 18, pp. 2818-2824, 2014.

[42] Z. L. Wang, "On the first principle theory of nanogenerators from Maxwell's equations," Nano Energy, vol. 68, article 104272, 2019.

[43] Z. L. Wang, "Triboelectric nanogenerator (TENG)-sparking an energy and sensor revolution," Advanced Energy Materials, vol. 10, no. 17, article 2000137, 2020.

[44] P. J. Landrigan, R. Fuller, N. J. R. Acosta et al., "The Lancet Commission on pollution and health," Lancet, vol. 391, no. 10119, pp. 462-512, 2018.

[45] S. O. Ganiyu, C. A. Martínez-Huitle, and M. A. Rodrigo, "Renewable energies driven electrochemical wastewater/soil decontamination technologies: a critical review of fundamental concepts and applications," Applied Catalysis B: Environmental, vol. 270, article 118857, 2020.

[46] B. P. Chaplin, "The prospect of electrochemical technologies advancing worldwide water treatment," Accounts of Chemical Research, vol. 52, no. 3, pp. 596-604, 2019.

[47] X. Cao, Y. Jie, N. Wang, and Z. L. Wang, "Triboelectric nanogenerators driven self-powered electrochemical processes for energy and environmental science," Advanced Energy Materials, vol. 6, no. 23, article 1600665, 2016.

[48] S. Bolisetty, M. Peydayesh, and R. Mezzenga, "Sustainable technologies for water purification from heavy metals: review and analysis," Chemical Society Reviews, vol. 48, no. 2, pp. 463-487, 2019.

[49] Z. Li, J. Chen, H. Guo et al., “Triboelectrification-enabled selfpowered detection and removal of heavy metal ions in wastewater," Advanced Materials, vol. 28, no. 15, pp. 2983-2991, 2016.

[50] L. Zhou, D. Liu, S. Li et al., "Effective removing of hexavalent chromium from wasted water by triboelectric nanogenerator driven self-powered electrochemical system - Why pulsed DC is better than continuous DC?," Nano Energy, vol. 64, article 103915, 2019.

[51] Z. Li, J. Chen, J. Yang et al., “ $\beta$-cyclodextrin enhanced triboelectrification for self-powered phenol detection and electrochemical degradation," Energy \& Environmental Science, vol. 8, no. 3, pp. 887-896, 2015.

[52] S. Gao, J. Su, X. Wei et al., "Self-powered electrochemical oxidation of 4 -aminoazobenzene driven by a triboelectric nanogenerator," ACS Nano, vol. 11, no. 1, pp. 770-778, 2017.

[53] S. Gao, Y. Chen, J. Su et al., "Triboelectric nanogenerator powered electrochemical degradation of organic pollutant using Pt-free carbon materials," ACS Nano, vol. 11, no. 4, pp. 3965-3972, 2017.

[54] S. Chen, N. Wang, L. Ma et al., "Triboelectric nanogenerator for sustainable wastewater treatment via a self-powered 
electrochemical process," Advanced Energy Materials, vol. 6, no. 8, article 1501778, 2016.

[55] Y. Yang, H. Zhang, S. Lee, D. Kim, W. Hwang, and Z. L. Wang, "Hybrid energy cell for degradation of methyl orange by self-powered electrocatalytic oxidation," Nano Letters, vol. 13, no. 2, pp. 803-808, 2013.

[56] S. Gao, M. Wang, Y. Chen et al., "An advanced electroFenton degradation system with triboelectric nanogenerator as electric supply and biomass-derived carbon materials as cathode catalyst," Nano Energy, vol. 45, pp. 2127, 2018.

[57] M. Tian, Y. Zhu, D. Zhang et al., "Pyrrolic-nitrogen-rich biomass-derived catalyst for sustainable degradation of organic pollutant _via_ a self-powered electro-Fenton process," Nano Energy, vol. 64, article 103940, 2019.

[58] Y. Chen, Y. Zhu, M. Tian, C. Chen, X. Jia, and S. Gao, "Sustainable self-powered electro-Fenton degradation of organic pollutants in wastewater using carbon catalyst with controllable pore activated by EDTA-2Na," Nano Energy, vol. 59, pp. 346-353, 2019.

[59] Y. Feng, K. Han, T. Jiang et al., "Self-powered electrochemical system by combining Fenton reaction and active chlorine generation for organic contaminant treatment," Nano Research, vol. 12, no. 11, pp. 2729-2735, 2019.

[60] M. Tian, D. Zhang, M. Wang et al., "Engineering flexible 3D printed triboelectric nanogenerator to self-power electroFenton degradation of pollutants," Nano Energy, vol. 74, article 104908, 2020.

[61] Q. Jiang, Y. Jie, Y. Han et al., "Self-powered electrochemical water treatment system for sterilization and algae removal using water wave energy," Nano Energy, vol. 18, pp. 81-88, 2015.

[62] R. E. Frye, J. Cakir, S. Rose et al., "Prenatal air pollution influences neurodevelopment and behavior in autism spectrum disorder by modulating mitochondrial physiology," Molecular Psychiatry, 2020.

[63] H. Guo, J. Chen, L. Wang et al., "A highly efficient triboelectric negative air ion generator," Nature Sustainability, vol. 4, no. 2, pp. 147-153, 2021.

[64] Y. Z. Liu, R. T. Guo, C. P. Duan et al., "Removal of gaseous pollutants by using 3DOM-based catalysts: a review," Chemosphere, vol. 262, article 127886, 2021.

[65] S. Chen, C. Gao, W. Tang et al., "Self-powered cleaning of air pollution by wind driven triboelectric nanogenerator," Nano Energy, vol. 14, pp. 217-225, 2015.

[66] K. Han, J. Luo, Y. Feng et al., "Wind-driven radial-engineshaped triboelectric nanogenerators for self-powered absorption and degradation of $\mathrm{NO}_{\mathrm{X}}$," ACS Nano, vol. 14, no. 3, pp. 2751-2759, 2020.

[67] Y. Feng, L. Ling, J. Nie et al., "Self-powered electrostatic filter with enhanced photocatalytic degradation of formaldehyde based on built-in triboelectric nanogenerators," ACS Nano, vol. 11, no. 12, pp. 12411-12418, 2017.

[68] S. Fukuzumi, "Production of liquid solar fuels and their use in fuel cells," Joule, vol. 1, no. 4, pp. 689-738, 2017.

[69] P. Gao, L. Zhang, S. Li, Z. Zhou, and Y. Sun, "Novel heterogeneous catalysts for $\mathrm{CO} 2$ hydrogenation to liquid fuels," ACS Central Science, vol. 6, no. 10, pp. 1657-1670, 2020.

[70] S.-F. Leung, H.-C. Fu, M. Zhang et al., "Blue energy fuels: converting ocean wave energy to carbon-based liquid fuels via $\mathrm{CO} 2$ reduction," Energy \& Environmental Science, vol. 13, no. 5, pp. 1300-1308, 2020.

[71] Q. Jiang, Y. Han, W. Tang et al., "Self-powered seawater desalination and electrolysis using flowing kinetic energy," Nano Energy, vol. 15, pp. 266-274, 2015.

[72] X. Ren, H. Fan, C. Wang et al., "Wind energy harvester based on coaxial rotatory freestanding triboelectric nanogenerators for self-powered water splitting," Nano Energy, vol. 50, pp. 562-570, 2018.

[73] A. Wei, X. Xie, Z. Wen et al., "Triboelectric nanogenerator driven self-powered photoelectrochemical water splitting based on hematite photoanodes," ACS Nano, vol. 12, no. 8, pp. 8625-8632, 2018.

[74] W. Tang, Y. Han, C. B. Han, C. Z. Gao, X. Cao, and Z. L. Wang, "Self-powered water splitting using flowing kinetic energy," Advanced Materials, vol. 27, no. 2, pp. 272-276, 2015.

[75] T. Li, Y. Xu, F. Xing et al., "Boosting photoelectrochemical water splitting by TENG-charged Li-ion battery," Advanced Energy Materials, vol. 7, no. 15, article 1700124, 2017.

[76] Y. Yang, H. Zhang, Z.-H. Lin et al., "A hybrid energy cell for self-powered water splitting," Energy \& Environmental Science, vol. 6, no. 8, article 2429, 2013.

[77] W. H. Wang, Y. Himeda, J. T. Muckerman, G. F. Manbeck, and E. Fujita, " $\mathrm{CO}_{2}$ hydrogenation to formate and methanol as an alternative to photo- and electrochemical $\mathrm{CO}_{2}$ reduction," Chemical Reviews, vol. 115, no. 23, pp. 12936-12973, 2015.

[78] N. Zhai, Z. Wen, X. Chen et al., "Blue energy collection toward all-hours self-powered chemical energy conversion," Advanced Energy Materials, vol. 10, no. 33, article 2001041, 2020.

[79] G. Duan, Y. Chen, Y. Tang et al., "Advances in electrocatalytic ammonia synthesis under mild conditions," Progress in Energy and Combustion Science, vol. 81, article 100860, 2020.

[80] A. Chen and B. Y. Xia, "Ambient dinitrogen electrocatalytic reduction for ammonia synthesis," Journal of Materials Chemistry A, vol. 7, no. 41, pp. 23416-23431, 2019.

[81] S. Gao, Y. Zhu, Y. Chen et al., "Self-power electroreduction of $\mathrm{N}_{2}$ into $\mathrm{NH}_{3}$ by 3D printed triboelectric nanogenerators," Materials Today, vol. 28, pp. 17-24, 2019.

[82] K. Han, J. Luo, Y. Feng, L. Xu, W. Tang, and Z. L. Wang, "Self-powered electrocatalytic ammonia synthesis directly from air as driven by dual triboelectric nanogenerators," Energy \& Environmental Science, vol. 13, no. 8, pp. 24502458, 2020.

[83] H. Zhu, Y. Xu, Y. Han et al., "Self-powered electrochemical anodic oxidation: a new method for preparation of mesoporous $\mathrm{Al}_{2} \mathrm{O}_{3}$ without applying electricity," Nano Research, vol. 8, no. 11, pp. 3604-3611, 2015.

[84] G. Zhu, C. Pan, W. Guo et al., "Triboelectric-generatordriven pulse electrodeposition for micropatterning," Nano Letters, vol. 12, no. 9, pp. 4960-4965, 2012.

[85] X. Zheng, J. Su, X. Wei, T. Jiang, S. Gao, and Z. L. Wang, "Self-powered electrochemistry for the oxidation of organic molecules by a cross-linked triboelectric nanogenerator," Advanced Materials, vol. 28, no. 26, pp. 5188-5194, 2016.

[86] J. Wang, Z. Wen, Y. Zi et al., "Self-powered electrochemical synthesis of polypyrrole from the pulsed output of a triboelectric nanogenerator as a sustainable energy system," 
Advanced Functional Materials, vol. 26, no. 20, pp. 3542$3548,2016$.

[87] S. Wang, L. Lin, and Z. L. Wang, "Triboelectric nanogenerators as self-powered active sensors," Nano Energy, vol. 11, pp. 436-462, 2015.

[88] H. Zhang, Y. Yang, T.-C. Hou, Y. Su, C. Hu, and Z. L. Wang, "Triboelectric nanogenerator built inside clothes for selfpowered glucose biosensors," Nano Energy, vol. 2, no. 5, pp. 1019-1024, 2013.

[89] Y. Bai, L. Xu, C. He et al., "High-performance triboelectric nanogenerators for self-powered, in-situ and real-time water quality mapping," Nano Energy, vol. 66, article 104117, 2019.

[90] H. Zhang, Y. Yang, Y. Su et al., "Triboelectric nanogenerator as self-powered active sensors for detecting liquid/gaseous water/ethanol," Nano Energy, vol. 2, no. 5, pp. 693-701, 2013.

[91] Y. N. X. Z. H. Lin, Y. Yang, S. H. Wang, G. Zhu, and Z. L. Wang, "Enhanced triboelectric nanogenerators and triboelectric nanosensor using chemically modified $\mathrm{TiO}_{2}$ nanomaterials," ACS Nano, vol. 7, no. 5, pp. 4554-4560, 2013.

[92] Y. Wu, Y. Su, J. Bai et al., "A self-powered triboelectric nanosensor for PH detection," Journal of Nanomaterials, vol. 2016, Article ID 5121572, 6 pages, 2016.

[93] Z. H. Lin, G. Zhu, Y. S. Zhou et al., "A self-powered triboelectric nanosensor for mercury ion detection," Angewandte Chemie-International Edition, vol. 52, no. 19, pp. 50655069, 2013.

[94] N. W. Y. Jie, X. Cao, Y. Xu, T. Li, X. J. Zhang, and Z. L. Wang, "Self-powered triboelectric nanosensor with poly(tetrafluoroethylene) nanoparticle arrays for dopamine detection," ACS Nano, vol. 9, no. 8, pp. 8376-8383, 2015.

[95] Z. Wen, J. Chen, M.-H. Yeh et al., "Blow-driven triboelectric nanogenerator as an active alcohol breath analyzer," Nano Energy, vol. 16, pp. 38-46, 2015.

[96] G. Cai, J. Wang, and P. S. Lee, "Next-generation multifunctional electrochromic devices," Accounts of Chemical Research, vol. 49, no. 8, pp. 1469-1476, 2016.

[97] M. Zhang, Y. Jie, X. Cao et al., "Robust design of unearthed single-electrode TENG from three-dimensionally hybridized copper/polydimethylsiloxane film," Nano Energy, vol. 30, pp. 155-161, 2016.

[98] J.-G. Sun, T. N. Yang, I. S. Kuo, J.-M. Wu, C.-Y. Wang, and L.-J. Chen, "A leaf-molded transparent triboelectric nanogenerator for smart multifunctional applications," Nano Energy, vol. 32, pp. 180-186, 2017.

[99] M. H. Yeh, L. Lin, P. K. Yang, and Z. L. Wang, "Motiondriven electrochromic reactions for self-powered smart window system," ACS Nano, vol. 9, no. 5, pp. 4757-4765, 2015.

[100] X. Yang, G. Zhu, S. Wang et al., "A self-powered electrochromic device driven by a nanogenerator," Energy \& Environmental Science, vol. 5, no. 11, pp. 9462-9466, 2012.

[101] W. Qiu, Y. Feng, N. Luo, S. Chen, and D. Wang, "Sandwichlike sound-driven triboelectric nanogenerator for energy harvesting and electrochromic based on Cu foam," Nano Energy, vol. 70, article 104543, 2020.

[102] C. F. Sabel and D. G. Victor, "Governing global problems under uncertainty: making bottom-up climate policy work," Climatic Change, vol. 144, no. 1, pp. 15-27, 2015.

[103] C.-Q. Ye, R.-G. Hu, S.-G. Dong et al., "EIS analysis on chloride-induced corrosion behavior of reinforcement steel in simulated carbonated concrete pore solutions," Journal of Electroanalytical Chemistry, vol. 688, pp. 275-281, 2013.

[104] M. M. S. Cheung and C. Cao, "Application of cathodic protection for controlling macrocell corrosion in chloride contaminated RC structures," Construction and Building Materials, vol. 45, pp. 199-207, 2013.

[105] J. Xu and W. Yao, "Current distribution in reinforced concrete cathodic protection system with conductive mortar overlay anode," Construction and Building Materials, vol. 23, no. 6, pp. 2220-2226, 2009.

[106] V. T. N. C. L. Page and M. M. Page, "Corrosion inhibitors in concrete repair systems," Magazine of Cocrete Research, vol. 52, no. 1, pp. 25-37, 2000.

[107] S. Cui, Y. Zheng, J. Liang, and D. Wang, "Conducting polymer PPy nanowire-based triboelectric nanogenerator and its application for self-powered electrochemical cathodic protection," Chemical Science, vol. 7, no. 10, pp. 6477-6483, 2016.

[108] Y. Feng, Y. Zheng, Z. U. Rahman, D. Wang, F. Zhou, and W. Liu, "Paper-based triboelectric nanogenerators and their application in self-powered anticorrosion and antifouling," Journal of Materials Chemistry A, vol. 4, no. 46, pp. 1802218030, 2016.

[109] S. Cui, Y. Zheng, J. Liang, and D. Wang, "Triboelectrification based on double-layered polyaniline nanofibers for selfpowered cathodic protection driven by wind," Nano Research, vol. 11, no. 4, pp. 1873-1882, 2018.

[110] H. R. Zhu, W. Tang, C. Z. Gao et al., "Self-powered metal surface anti-corrosion protection using energy harvested from rain drops and wind," Nano Energy, vol. 14, pp. 193-200, 2015.

[111] W. Guo, X. Li, M. Chen et al., "Electrochemical cathodic protection powered by triboelectric nanogenerator," Advanced Functional Materials, vol. 24, no. 42, pp. 6691-6699, 2014.

[112] Z. Wang, L. Cheng, Y. Zheng, Y. Qin, and Z. L. Wang, "Enhancing the performance of triboelectric nanogenerator through prior-charge injection and its application on selfpowered anticorrosion," Nano Energy, vol. 10, pp. 37-43, 2014.

[113] X. Li, J. Tao, W. Guo et al., "A self-powered system based on triboelectric nanogenerators and supercapacitors for metal corrosion prevention," Journal of Materials Chemistry A, vol. 3, no. 45, pp. 22663-22668, 2015.

[114] L. Zhou, D. Liu, Z. Zhao et al., "Simultaneously enhancing power density and durability of sliding-mode triboelectric nanogenerator via interface liquid lubrication," Advanced Energy Materials, vol. 10, no. 45, article 2002920, 2020.

[115] M. Ingelsson, N. Yasri, and E. P. L. Roberts, "Electrode passivation, faradaic efficiency, and performance enhancement strategies in electrocoagulation-a review," Water Research, vol. 187, article 116433, 2020. 